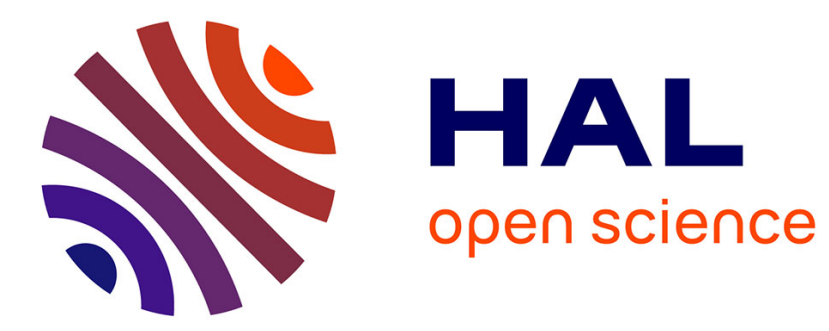

\title{
Testing for Leverage Effects in the Returns of US Equities
}

Christophe Chorro, Dominique Guegan, Florian Ielpo, Hanjarivo Lalaharison

\section{To cite this version:}

Christophe Chorro, Dominique Guegan, Florian Ielpo, Hanjarivo Lalaharison. Testing for Leverage Effects in the Returns of US Equities. 2017. halshs-00973922v2

\section{HAL Id: halshs-00973922 \\ https://shs.hal.science/halshs-00973922v2}

Submitted on 19 Jan 2017

HAL is a multi-disciplinary open access archive for the deposit and dissemination of scientific research documents, whether they are published or not. The documents may come from teaching and research institutions in France or abroad, or from public or private research centers.
L'archive ouverte pluridisciplinaire HAL, est destinée au dépôt et à la diffusion de documents scientifiques de niveau recherche, publiés ou non, émanant des établissements d'enseignement et de recherche français ou étrangers, des laboratoires publics ou privés. 


\section{Documents de Travail du PANTHÉN SORBONNE Centre d'Economie de la Sorbonne}
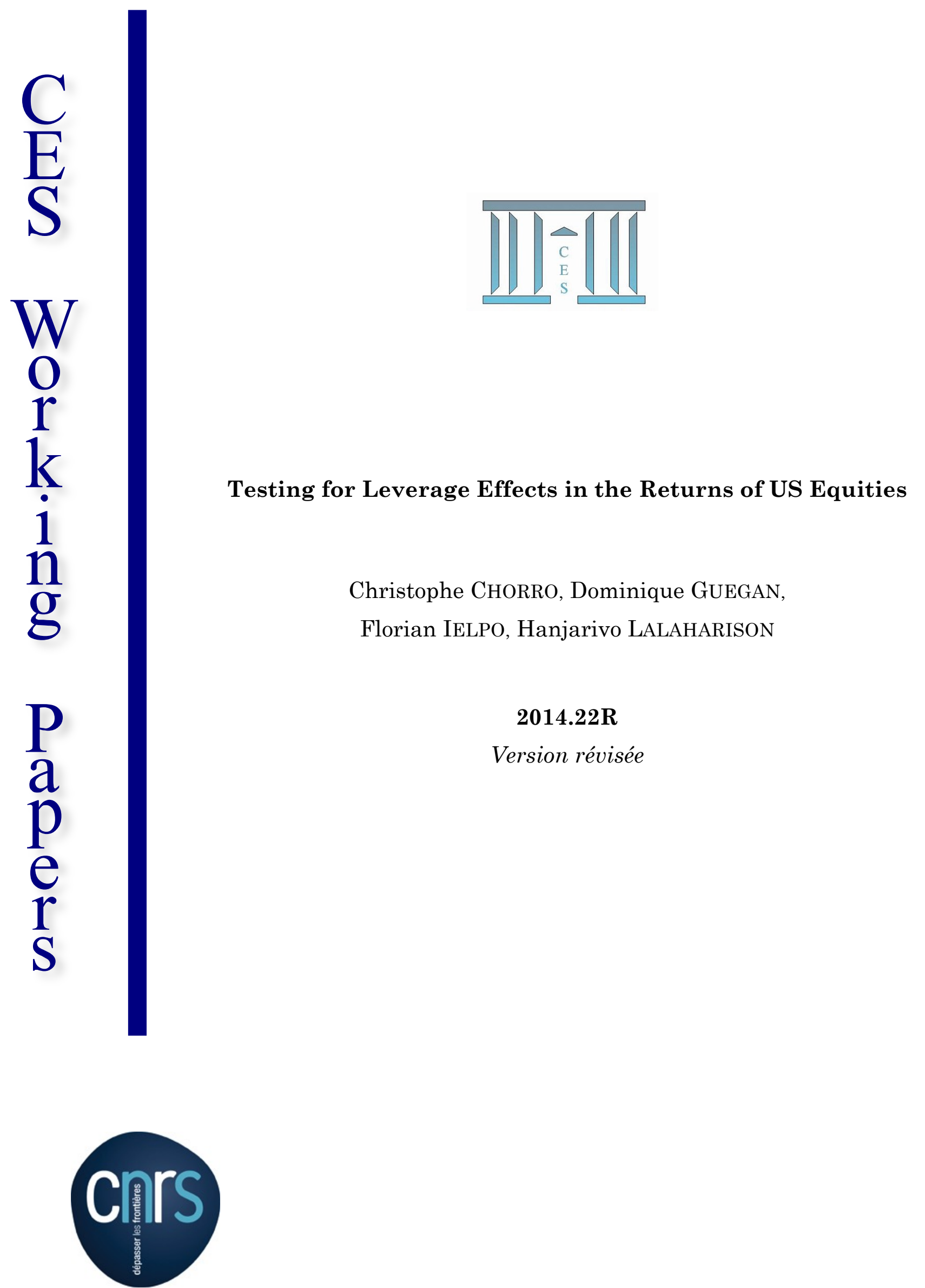

Maison des Sciences Économiques, 106-112 boulevard de L'Hôpital, 75647 Paris Cedex 13 http://centredeconomiesorbonne.univ-paris $1 . \mathrm{fr} /$ 


\title{
Testing for Leverage Effects in the Returns of US Equities
}

\author{
Christophe Chorro* $\quad$ Dominique Guégan ${ }^{\dagger} \quad$ Florian Ielpo $^{\ddagger} \quad$ Hanjarivo Lalaharison ${ }^{\S}$
}

December 22, 2016

\begin{abstract}
This article questions the empirical usefulness of leverage effects to describe the dynamics of equity returns. Relying on both in and out of sample tests we consistently find a weak contribution of leverage effects over the past 25 years of S\&P 500 returns. The skewness in the conditional distribution of the returns's time series model is found to explain most of the returns' distribution's asymmetry. This conclusion holds both at the index level and for $70 \%$ of the individual stocks constituents of the equity index. ${ }^{1}$
\end{abstract}

Keywords: Asymmetry, GARCH, Mixture of Gaussian distributions, Generalized hyperbolic distributions, S\&P 500, Leverage effect.

JEL classification: C12, C22, G17.

\section{Introduction}

An accurate description of the dynamics of stock index returns requires a mix between a time varying volatility structure and an asymmetric and fat-tailed distribution. That is the conclusion of a long stream of research papers published over the past decades. The time varying volatility structure accounts for the potential changes in the level of risk in financial markets when the conditional distribution deals with the rare occurrence of extreme events, usually seen as jumps. Such an approach turned out to be both successful when applied in continuous - as in Bates (1996)'s extension of Heston (1993)'s work - and in discrete time models - as in Christoffersen et al. (2006, 2010), Badescu et al. (2008), Chorro et al. (2010, 2012) or Guégan et al. (2013). The estimation of such models is a complex matter as components in the volatility structure and in the conditional distribution can impact the model implied density in a very similar way. The asymmetric past return to volatility feedback effect in discrete time models usually refereed to as "leverage effect" can have a similar impact to that of the asymmetry in the returns' conditional distribution. Building on a recursive numerical implementation of the maximum-likelihood estimation presented in the Section 2.6.3 of Chorro et

\footnotetext{
*University Paris 1 Panthéon-Sorbonne, Centre d'Economie de la Sorbonne and Labex ReFi, 106 bd de l'hopital, 75013, Paris, France. Email: cchorro@univ-paris1.fr. Tel: +331440782 78.

${ }^{\dagger}$ University Paris 1 Panthéon-Sorbonne, CES and Labex ReFi, 106 bd de 1'hopital, 75013, Paris, France. Email: dguegan@univ-paris1.fr.

${ }^{\ddagger}$ Unigestion, Centre d’Economie de la Sorbonne and Ipag Business School. Email: fielpo@unigestion.com.

${ }^{\S}$ Université d'Antananarivo, Faculté des sciences, Mention Mathématiques et Informatique. Email: lalaharisonh@gmail.com.

${ }^{1}$ This work was achieved through the Laboratory of Excellence on Financial Regulation (Labex ReFi) supported by PRES heSam under the reference ANR10LABX0095. It benefited from a French government support managed by the National Research Agency (ANR) within the project Investissements d'Avenir Paris Nouveaux Mondes (investments for the future Paris New Worlds) under the reference ANR11IDEX000602. The authors thank the participants to the following conferences: CFE 2013 in London, FEBS 2014 hosted by the Surrey University, the IAAE 2014 in London, the EEA-ESEM 2016 in Geneva Switzerland and the Paris Financial Management Conference 2016 in Paris.
} 
al. (2016), this article aims at disentangling both effects and then assess which of the two explains the largest share of the returns on stocks' skewness. Our focus here is on a dataset of returns on US stocks, the most commonly used dataset in financial econometrics empirical experiments. Our results suggest that leverage effects have actually been negligible over the past 25 years of returns history.

Leverage effects consist in an asymmetric reaction of volatility to past returns, volatility rising more rapidly when returns are negative than positive (see Aydemir et al., 2006). This stylized fact first highlighted in Black (1976) and Christie (1982) is usually explained in two ways: first, an increase in volatility should coincide with a higher expected return - as prices drop - when the price of risk is constant. Second, a drop in the price of a stock increases the underlying company's financial leverage and the volatility of its stock increases as a response to this increase in the firm's risk. Nevertheless, the lack of consensus around the rationale to leverage effect is important as illustrated by the various contributions of Schwert (1989), Campbell and Hentschel (1992), Duffee (1995), Bekaert and Wu (2000), Figlewski and Wang (2000), Wu (2001), Aydemir et al. (2006) and Bae et al. (2007). Regardless of the conclusions raised in each of these articles regarding the economic origin of the phenomenon, they unanimously acknowledged the empirical existence of leverage effects in individual stocks. Now, when aggregating these firm-specific phenomenon into equity indices, it is unsure whether the volatility feedback or the financial argument hold as idiosyncratic risk is getting diversified. Still, a significant number of articles maintain an asymmetric component in their modeling of volatility usually in order to generate time varying negative skewness. Examples of these contributions are Poon and Granger (2003), Awartani and Corradi (2005), Corsi and Reno (2012), Bandi and Reno (2012) and Hansen et al. (2012). In the continuous time literature, a similar case can be made out of various extensions of the Heston (1993)'s model where the leverage effect component is used to generate asymmetric implied volatility surfaces as extracted from option prices. Leverage effects probably turned out to be a useful component improving continuous and discrete time financial models' ability to fit the observed distribution of returns (Awartani and Corradi, 2005) or to match the observed price of options on stock indices (Christoffersen and Jacobs, 2004) especially when the model's conditional distribution is Gaussian and thus does not incorporate any asymmetry or fat tails. Alternatively, a properly selected conditional distribution can provide an interesting goodness-of-fit of the returns' distribution as well (see e.g. Wang et al., 2001. Giot and Laurent (2004) and Curto et al., 2009), somewhat in a very comparable way to that of the leverage effect.

Thus, the theoretical economic reliability of leverage effects in the dynamics of returns on indices and the possibility to use alternative flexible distributions cast doubts on the necessity of a leverage component in conditional volatility structures. This article aims to provide a reliable measure of it using 25 years of S\&P 500 returns. Following Badescu et al. $(2008,2011)$ and Chorro et al. (2010, 2012), we combine two classical asymmetric GARCH specifications used in the financial literature, namely, the Exponential GARCH (EGARCH) introduced by Nelson (1991) and the Asymmetric Power ARCH model (APARCH) of Ding et al. (1993), with two families of conditional distributions that are able to generate various levels of skewness and kurtosis: the Generalized Hyperbolic distribution as introduced by Barndorff-Nielsen (1977) and the mixture of two Gaussian distributions (see e.g. Behboodian, 1970). By Combining these two components we want to be able to disentangle the part of the skewness effectively coming from the leverage effect and the part coming from the left tail of the conditional distribution.

We both test the statistical significance of leverage in the dynamics of the S\&P 500's returns based on in- and out-of-sample approaches. Our conclusions unfold as follows: first, we find that leverage effects only account for 10 to $20 \%$ of the returns' total skewness, the rest of it being driven by the asymmetry of the conditional distribution. Second, by performing in-sample Hansen (1992)'s test, we consistently accept the hypothesis that the parameter driving the leverage effect in the asymmetric 
GARCH models can be set to zero. Finally, we consistently find that leverage effects do not statistically improve the out-of-sample forecast accuracy of our time series models when using the Amisano and Giacomini (2007)'s test methodology when the conditional distribution is asymmetric. All results are stable when splitting the sample to check for the robustness of our findings. Hence, our results indicate that modeling the S\&P 500's returns through an asymmetric GARCH model is not statistically relevant as long as the conditional distribution is flexible enough to capture the behavior of the returns' distribution's tails. Finally, when testing for leverage effects on the components of the S\&P 500 index, we find that $70 \%$ of stocks do not exhibit a statistically significant leverage effect parameter.

The article is organized as follows. Section 2 presents the models and the tests used to detect or not leverage effects. Section 3 details the empirical results. Section 4 concludes.

\section{Methodology}

In this section we present the models we used in the empirical part of this article and the Hansen's test and the Amisano and Giacomini's test.

\subsection{Modeling S\&P500 log-returns with asymmetric GARCH processes and non-Gaussian innovations}

Let $\left(Y_{t}\right)_{t \in\{1, \ldots, T\}}$ be a sequence of random variables, defined on a probability space $(\Omega, \mathcal{A}, \mathbb{P})$, representing the daily log-returns of the S\&P500 index that we model through a class of related $\operatorname{GARCH}(1,1)$ processes:

$$
\begin{gathered}
Y_{t}=\sqrt{h_{t}} z_{t}, \quad z_{0}=x \in \mathbb{R}, \\
h_{t}=F_{\theta^{V}}\left(z_{t-1}, h_{t-1}\right)
\end{gathered}
$$

where $\left(z_{t}\right)_{t \in\{1, \ldots, T\}}$ is a sequence of independent and identically distributed real random variables with a density $d_{\theta^{D}}$ that depends on a set of parameters $\theta^{D}$ and where $F_{\theta^{V}}$ indexed by a set of parameters $\theta^{V}$ models the volatility in the following way:

1. First using the Nelson (1991)'s EGARCH model

$$
\log \left(h_{t}\right)=\alpha_{0}+\alpha_{1}\left|z_{t-1}\right|+\gamma z_{t-1}+\beta_{1} \log \left(h_{t-1}\right),
$$

2. Second using the Ding et al. (1993)'s APARCH model

$$
h_{t}^{\delta_{v o l}}=\alpha_{0}+\alpha_{1}\left(\left|z_{t-1}\right|-\gamma z_{t-1}\right)^{\delta_{v o l}}+\beta_{1} h_{t-1}^{\delta_{v o l}} .
$$

These models are chosen for their ability to generate not only time varying volatility but also leverage effects by means of the parameter $\gamma$. For the residuals we use two classes of distributions: (i) a mixture of two Gaussian distributions ${ }^{2}$ (Kon (1984), Akgiray and Booth (1987), Tucker and Pond

\footnotetext{
${ }^{2}$ The mixture of two Gaussian distributions has the density

$$
f(x)=\Phi f\left(x, \mu_{1}, \sigma_{1}\right)+(1-\Phi) f\left(x, \mu_{2}, \sigma_{2}\right),
$$

where $\left(\phi, \mu_{1}, \mu_{2}, \sigma_{1}, \sigma_{2}\right) \in[0,1] \times \mathbb{R}^{2} \times\left(\mathbb{R}^{*}\right)^{2}$ and where $f\left(., \mu_{i}, \sigma_{i}\right)$ is the density of a Gaussian random variable with expectation $\mu_{i}$ and standard deviation $\sigma_{i}$.
} 
(1998) and Alexander and Lazar (2006)); (ii) the Generalized Hyperbolic distribution ${ }^{3}$ (BarndorffNielsen (1977)). For both choices, the number of parameters driving the distribution is five. Adding the parameters involved in the different GARCH specifications we use, the estimation of such models leads to important optimization issues due to the dimension of the problem (see for example Fan et al. (2015)). In this paper we use a numerical implementation of the so-called conditional maximumlikelihood estimation that has been recently introduced and backtested in Chorro et al. (2016), Section 2.6.3. This empirical recursive methodology, that starts from the Quasi maximum-likelihood estimate and that iteratively maximizes the likelihood over either the volatility or the distributions parameters, is given in the Appendix.

\subsection{Testing methodologies for the significance of leverage effects}

We introduce now the two tests we use in the empirical part to test significance or not of the leverage effect parameter $\gamma$. First we test the in-sample significance of the leverage component in GARCHtype models using Hansen (1992)'s test. Second, we confirm the findings obtained using the previous step by gauging the usefulness of the leverage effect parameter when it comes to forecasting the future density of returns. To do so, we rely on Amisano and Giacomini (2007)'s forecast density test methodology.

To test for the significance of the leverage parameter in the various GARCH specifications considered previously we consider the in-sample performances of GARCH type models with or without leverage parameters applying the methodology developed by Hansen (1992). We consider a sample of size $T$ $\left(y_{1}, \ldots, y_{T}\right)$ for the log-returns.

In this part we denote by $\gamma \in \Gamma$ the leverage parameter of the volatility structures we consider (see equations (3) and (4)) and by $\theta \in \Theta$ all the remaining parameters. The null and alternative hypotheses are:

$$
H_{0}: \gamma=0 \quad \text { vs } \quad H_{1}: \gamma \neq 0 .
$$

We use as a test's statistics, the concentrated and standardized likelihood ratio statistics

$$
\hat{L R}_{T}^{*}=\sup _{\gamma \in \Gamma} \frac{\hat{L R_{T}(\gamma)}}{V_{T}(\gamma)^{1 / 2}},
$$

with $\hat{L R_{T}}(\gamma)=L_{T}\left(\gamma, \hat{\theta}_{T}(\gamma)\right)-L_{T}\left(0, \hat{\theta}_{T}(0)\right)$ where

$$
L_{T}(\gamma, \theta)=\sum_{i=1}^{T} l_{i}(\gamma, \theta)
$$

$l_{i}(\gamma, \theta)$ being the conditional $\log$-likelihood given $\mathcal{F}_{i-1}$, and where

$$
\hat{\theta}_{T}(\gamma)=\operatorname{argmax}_{\theta \in \Theta} L_{T}(\gamma, \theta)
$$

${ }^{3}$ For $(\lambda, \alpha, \beta, \delta, \mu) \in \mathbb{R}^{5}$ with $\delta>0$ and $\alpha>|\beta|>0$, the density function is defined in this case by

$$
f(x)=\frac{\left(\sqrt{\alpha^{2}-\beta^{2}} / \delta\right)^{\lambda}}{\sqrt{2 \pi} K_{\lambda}\left(\delta \sqrt{\alpha^{2}-\beta^{2}}\right)} e^{\beta(x-\mu)} \frac{K_{\lambda-1 / 2}\left(\alpha \sqrt{\delta^{2}+(x-\mu)^{2}}\right)}{\left(\sqrt{\delta^{2}+(x-\mu)^{2}} / \alpha\right)^{1 / 2-\lambda}}
$$

where $K($.$) is the modified Bessel function of the third kind.$ 
and

$$
V_{T}(\gamma)=\sum_{i=1}^{T}(\underbrace{l_{i}\left(\gamma, \hat{\theta}_{T}(\gamma)\right)-l_{i}\left(0, \hat{\theta}_{T}(0)\right)-\frac{\hat{L R_{T}(\gamma)}}{T}}_{q_{i}\left(\gamma, \hat{\theta}_{T}(\gamma)\right)})^{2} .
$$

We don't have an asymptotic distribution for $\hat{L R_{T}^{*}}$ but an asymptotic upper bound for the tail function useful for the computation of the $\mathrm{p}$ value of the test:

$$
\mathbb{P}\left(\hat{L R_{T}^{*}} \geq x\right) \leq B_{T}(x) \underset{T \rightarrow \infty}{\longrightarrow} \mathbb{P}\left(\sup _{\gamma \in \Gamma} Q^{*}(\gamma) \geq x\right)
$$

where $Q^{*}$ is a centered Gaussian process with a covariance function $K^{*}$ that may be approximated for large $T$ by its sample counterpart

$$
\hat{K}_{T}^{*}\left(\gamma_{1}, \gamma_{2}\right)=\frac{\hat{K}_{T}\left(\gamma_{1}, \gamma_{2}\right)}{V_{T}\left(\gamma_{1}\right)^{1 / 2} V_{T}\left(\gamma_{2}\right)^{1 / 2}}
$$

with

$$
\hat{K}_{T}\left(\gamma_{1}, \gamma_{2}\right)=\sum_{i=1}^{T} \hat{q}_{i}\left(\gamma_{1}\right) \hat{q}_{i}\left(\gamma_{2}\right)+\sum_{k=1}^{M} w_{k, M}\left[\sum_{i=1}^{T-k} \hat{q}_{i}\left(\gamma_{1}\right) \hat{q}_{i+k}\left(\gamma_{2}\right)+\sum_{i=1+k}^{T} \hat{q}_{i}\left(\gamma_{1}\right) \hat{q}_{i-k}\left(\gamma_{2}\right)\right],
$$

where $\hat{q}_{i}(\gamma)=q_{i}\left(\gamma, \hat{\theta}_{T}(\gamma)\right)$ and $w_{k, M}=1-|k| /(M+1)$ is the so-called Bartlett kernel with $M$ as a bandwidth ${ }^{4}$.

To assess the contribution of leverage effects when it comes to returns forecasting we use the forecasting density test described in Amisano and Giacomini (2007). In our presentation we follow Maheu and McCurdy (2011), focusing on the ability of the approach to test multi-period forecasts.

For $R \in\{A, B\}$ we consider two competing related GARCH models ${ }^{5}$. Starting from the sample $\left(y_{1}, \ldots y_{T}\right)$ we test forecast horizons $1 \leq k \leq k_{\max }$ through rolling-window forecasting schemes of size $\tau$. According to Amisano Giacomini (2007), under the null hypothesis of equal predictive performances for both models, the statistic based on predictive likelihoods of horizon $k$ for models $A$ and $B$

$$
t_{A, B}^{k}=\frac{\left(D_{A, k}-D_{B, k}\right) \sqrt{T-\tau-k_{\max }+1}}{\hat{\sigma}_{A, B, k}}
$$

is asymptotically Gaussian. In equation (10),

${ }^{4}$ The approximated $\mathrm{p}$ values of the test can now be computed by Monte Carlo methods: when $\left(u_{i}\right)_{1 \leq i \leq T+M}$ are i.i.d $\mathcal{N}(0,1)$,

$$
\tilde{L R}^{*}(\gamma)=\frac{\sum_{k=0}^{M} \sum_{i=1}^{T} \hat{q}_{i}(\gamma) u_{i+k}}{\sqrt{1+M} V_{T}(\gamma)^{1 / 2}}
$$

is a Gaussian process with covariance $\hat{K}_{T}^{*}$. Moreover, we can compute its supremum over $\gamma$ forming a reasonable grid $\left[\gamma_{0}, \ldots, \gamma_{N}\right]$ centered on the value 0 . Practically, the theory does not give any particular guidance for the choice of $M$, thus, all the numerical work is calculated for $M=0,1, \ldots, 5$. We also consider a grid with step size 0.05 containing 10 points centered on 0 and in order to achieve some efficiency in the computation of $\tilde{L R}^{*}$ we compute along the grid each $\hat{\theta}_{T}(\gamma)$ using as a starting point the preceding obtained value. Then, the distribution of $\sup _{\gamma \in \Gamma} Q^{*}(\gamma)$ and the associated $\mathrm{p}$ values of the test are approximated using 1000 independent realizations of $\sup _{\gamma \in \Gamma} \tilde{L R}{ }^{*}(\gamma)$.

${ }^{5}$ In the empirical part, model $A$ is of the form (1), (2) depending on the leverage parameter $\gamma$ while model $B$ follows the same dynamics with $\gamma=0$. 


$$
D_{R, k}=\frac{1}{T-\tau-k_{\max }+1} \sum_{t=\tau+k_{\max }-k}^{T-k} \omega_{i}\left(y_{t+k}^{s d}\right) \log f_{R, k}\left(y_{t+k}, \widehat{\theta_{t+k}^{R}} \mid \mathcal{F}_{t}\right)
$$

where $\widehat{\theta_{t+k}^{R}}$ is the estimated vector of parameters for the model $R$ obtained using the sub-sample $\left(y_{t-\tau-k_{\text {max }}+1}, \ldots, y_{t}\right), f_{R, k}\left(y_{t+k}, \widehat{\theta_{t+k}^{R}} \mid \mathcal{F}_{t}\right)$ is the conditional density for the model $R$ of $Y_{t+k}$, given $\mathcal{F}_{t}$ and $\widehat{\theta_{t+k}^{R}}$, evaluated at the realized log-return $y_{t+k}$ and where $y_{t+k}^{s d}=\frac{y_{t+k}-\hat{\mu}_{k, t}}{\hat{\sigma}_{k, t}}$ is the standardized realized log-returns taking respectively for $\hat{\mu}_{k, t}$ and $\hat{\sigma}_{k, t}$ the mean and the standard deviation of $\left(y_{t-\tau-k_{\max }+1}, \ldots, y_{t}\right)$. Finally, $\hat{\sigma}_{A, B, k}$ is a properly selected estimator for the variance of

$$
\omega_{i}\left(y_{t+k}^{s d}\right)\left(\log f_{A, k}\left(y_{t+k}, \widehat{\theta_{t+k}^{A}} \mid \mathcal{F}_{t}\right)-\log f_{B, k}\left(y_{t+k}, \widehat{\theta_{t+k}^{B}} \mid \mathcal{F}_{t}\right)\right)
$$

where the weights $\omega_{i}$ are chosen to focus on particular regions of the forcasted distribution: (0) Whole distribution: $\omega_{0}(Y)=1$; (i) Center of distribution: $\omega_{1}(Y)=\phi(Y)$, $\phi$ standard normal density function; (ii) Tails of distribution: $\omega_{2}(Y)=1-\phi(Y) / \phi(0)$; (iii) Right tail: $\omega_{3}(Y)=\Phi(Y)$, $\Phi$ standard normal distribution function; (iv) Left tail: $\omega_{4}(Y)=1-\Phi(Y)$.

Here, as proposed Amisano Giacomini (2007), we use a Newey-West estimator for $\hat{\sigma}_{A, B, k}$ that take into account heteroskedasticity and autocorrelation. One of the main interest of this approach comes from the fact that the two models can be nested or not and can be estimated using very different techniques from the moment that they are based on a finite estimation window.

\section{Results}

This section is dedicated to the presentation of the results of our empirical experiments.

\subsection{Description of the data set}

We estimate the various models presented earlier using a data set of returns on the S\&P 500. The data set starts on January, $2^{\text {nd }} 1987$ and ends on July, 20 ${ }^{\text {st }} 2011$. It includes different market phases and four marked market crashes in 1987, 1998, 2001 and 2008. It is made of daily closing prices in US Dollar. The full sample contains 6190 observations. Figure 1 charts the evolution of the index over the sample. Descriptive statistics are provided by Table 1 . 


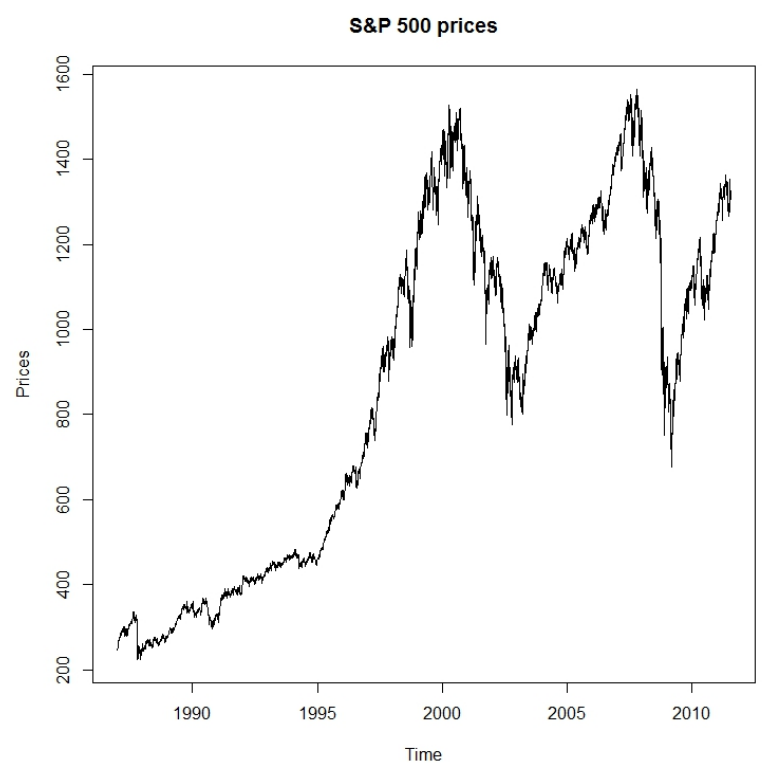

Figure 1: S\&P 500 prices. Data source: Bloomberg.

The data set starts on January, $2^{\text {nd }} 1987$ and ends on December, $31^{\text {st }}$ 2011. It includes 6190 daily data.

\begin{tabular}{|l|c|c|c|c|c|}
\hline \hline & Number of observations & Mean & Standard Deviation & Skewness & Kurtosis \\
\hline \hline S\&P 500 from 02/01/87 to 20/07/11 & 6190 & 0.027 & 0.190 & -1.341 & 29.075 \\
\hline S\&P 500 from 02/01/87 to 31/12/98 & 3034 & 0.052 & 0.164 & -3.936 & 84.788 \\
\hline S\&P 500 from 02/01/99 to 20/07/11 & 3156 & 0.002 & 0.212 & -0.115 & 7.412 \\
\hline \hline
\end{tabular}

Table 1: Descriptive statistics for the S\&P 500 daily log-returns.

This table presents the descriptive statistics for the three samples of the S\&P 500 log returns considered in the paper. The average and standard error statistics are annualized

The S\&P 500 returns are characterized by fat tails, as the excess kurtosis is positive in the full sample (29.075). The sample's skewness is equal to -1.341 highlighting the asymmetry to the left of the S\&P 500's returns's distribution. Both stylized facts are rather stable in the two sub-samples presented in Table 1. However, the skewness associated to the 1999-2011 sample is very close to zero. Investigating Figure 1, the balance between rising and falling phases explains part of this figure. Now, as discussed earlier, a zero skewness can be the result of the combination of leverage effects with positive conditional skewness, leaving ample room for our measurement experiments.

\subsection{The explanatory power of the leverage effect over US stocks' returns' skewness}

In this part, we simply report an estimate of the percentage of the sample's total skewness that can be accounted for by the leverage component of the GARCH-type models, once these models have been properly estimated using the recursive method detailed in the appendix part of this article. In order to measure the share of the sample's skewness that comes from the leverage effect and the share that comes from the conditional distribution, we rely on two sets of estimates. The first set comes from an estimation of EGARCH and APARCH models, mixed with a GH and a mixture of two 
Gaussian distributions with no restrictions on the parameters. A second set of estimates is obtained by constraining the parameter in the asymmetric GARCH models that control the leverage effect $\gamma$ (as in equations (3) and (4)) to be equal to zero. The metric that will help us to measure the importance of the leverage effect to explain the sample's skewness will be based on the skewness of the residuals obtains with each set of parameters for a given models. Let $s_{\gamma=0}$ be the skewness obtained in the residuals when $\gamma$ is constrained to zero and $s_{\gamma \neq 0}$ be the same quantity when $\gamma$ is unconstrained. The following ratio measures the decrease in the residuals' skewness that comes from the GARCH models' leverage component:

$$
r=\frac{s_{\gamma=0}-s_{\gamma \neq 0}}{s_{\gamma=0}}
$$

When the leverage component fully explains the returns' skewness, $s_{\gamma \neq 0}=0$ and $r=100 \%$. When this component does not explain any part of the sample's skewness, then $s_{\gamma \neq 0}=s_{\gamma=0}$ and $r=0$. Tables 2, 3 and 4 provide the parameters' estimates along with $r, s_{\gamma=0}$ and $s_{\gamma \neq 0}$ for each models and sub-samples of our main data set.

\section{EGARCH-GH}

\begin{tabular}{|c|c|c|c|c|c|c|c|c|c|c|c|}
\hline$\alpha$ & $\beta$ & $\delta$ & $\mu$ & $\lambda$ & $\alpha_{0}$ & $\gamma$ & $\alpha_{1}$ & $\beta_{1}$ & $s$ & $r$ & \\
\hline 0.164 & -0.163 & 1.836 & 0.142 & -2.885 & -0.222 & -0.058 & 0.118 & 0.985 & -1.112 & & \\
\hline$(0.075)$ & $(0.053)$ & $(0.267)$ & $(0.048)$ & $(0.350)$ & $(0.062)$ & $(0.013)$ & $(0.017)$ & $(0.006)$ & & & \\
\hline 0.259 & -0.136 & 1.719 & 0.128 & -2.484 & -0.174 & 0 & 0.113 & 0.990 & -1.481 & 0.249 & \\
\hline$(0.046)$ & $(0.032)$ & $(0.053)$ & $(0.025)$ & $(0.662)$ & $(0.083)$ & & $(0.024)$ & (0.007) & & & \\
\hline \multicolumn{12}{|c|}{ EGARCH-MN } \\
\hline$\Phi$ & $\mu_{1}$ & $\sigma_{1}$ & $\mu_{2}$ & $\sigma_{2}$ & $\alpha_{0}$ & $\gamma$ & $\alpha_{1}$ & $\beta_{1}$ & $s$ & $r$ & \\
\hline 0.047 & -0.863 & 2.228 & 0.038 & 0.836 & -0.275 & -0.068 & 0.138 & 0.981 & -1.034 & & \\
\hline (0.009) & $(0.251)$ & (1.012) & $(0.012)$ & $(0.325)$ & $(0.061)$ & $(0.013)$ & (0.019) & $(0.005)$ & & & \\
\hline 0.051 & -0.852 & 2.371 & 0.042 & 0.851 & -0.218 & 0 & 0.139 & 0.987 & -1.328 & 0.221 & \\
\hline$(0.012)$ & $(0.247)$ & (1.322) & $(0.015)$ & $(0.421)$ & $(0.046)$ & & $(0.015)$ & $(0.006)$ & & & \\
\hline \multicolumn{12}{|c|}{ APARCH-GH } \\
\hline$\alpha$ & $\beta$ & $\delta$ & $\mu$ & $\lambda$ & $\alpha_{0}$ & $\alpha_{1}$ & $\gamma$ & $\beta_{1}$ & $\delta_{\text {vol }}$ & $s$ & $r$ \\
\hline 0.137 & -0.136 & 1.824 & 0.125 & -2.758 & 0.000 & 0.091 & 0.597 & 0.907 & 1.037 & -0.884 & \\
\hline$(0.062)$ & $(0.035)$ & $(0.552)$ & $(0.046)$ & $(0.163)$ & $(0.0001)$ & $(0.018)$ & $(0.103)$ & $(0.022)$ & $(0.080)$ & & \\
\hline 0.158 & -0.157 & 1.807 & 0.146 & -2.721 & 0.000 & 0.161 & 0 & 0.944 & 1.252 & -1.417 & 0.376 \\
\hline$(0.022)$ & $(0.061)$ & $(0.609)$ & $(0.039)$ & $(0.745)$ & $(0.0001)$ & $(0.009)$ & & $(0.008)$ & $(0.067)$ & & \\
\hline \multicolumn{12}{|c|}{ APARCH-MN } \\
\hline$\Phi$ & $\mu_{1}$ & $\sigma_{1}$ & $\mu_{2}$ & $\sigma_{2}$ & $\alpha_{0}$ & $\alpha_{1}$ & $\gamma$ & $\beta_{1}$ & $\delta_{\text {vol }}$ & $s$ & $r$ \\
\hline 0.940 & 0.039 & 0.845 & -0.688 & 2.149 & 0.000 & 0.094 & 0.597 & 0.906 & 1.037 & -0.873 & \\
\hline$(0.021)$ & $(0.008)$ & $(0.213)$ & $(0.087)$ & (1.052) & $(0.000)$ & $(0.011)$ & $(0.082)$ & $(0.012)$ & $(0.120)$ & & \\
\hline 0.933 & 0.048 & 0.815 & -0.680 & 2.285 & 0.000 & 0.119 & 0 & 0.890 & 1.438 & -1.038 & 0.159 \\
\hline$(0.028)$ & $(0.016)$ & $(0.201)$ & $(0.085)$ & (1.112) & $(0.000)$ & $(0.013)$ & & (0.014) & $(0.173)$ & & \\
\hline
\end{tabular}

Table 2: Estimated parameters with and without leverage effect parameter using the S\&P 500 data set from January 2, 1987 to December 31, 1998 and their standard errors. $r=\frac{s_{\gamma=0}-s_{\gamma \neq 0}}{s_{\gamma=0}}$ where $s_{\gamma \neq 0}$ and $s_{\gamma=0}$ are the skewness of the residual with and without leverage effect respectively. 
EGARCH-GH

\begin{tabular}{|c|c|c|c|c|c|c|c|c|c|c|c|}
\hline \hline$\alpha$ & $\beta$ & $\delta$ & $\mu$ & $\lambda$ & $\alpha_{0}$ & $\gamma$ & $\alpha_{1}$ & $\beta_{1}$ & $s$ & $r$ & \\
\hline \hline 1.026 & -1.025 & 3.969 & 0.914 & -9.824 & -0.198 & -0.128 & 0.090 & 0.986 & -0.380 & & \\
$(0.226)$ & $(0.165)$ & $(0.387)$ & $(0.029)$ & $(1.471)$ & $(0.025)$ & $(0.011)$ & $(0.012)$ & $(0.002)$ & & & \\
1.834 & -0.299 & 1.536 & 0.191 & -0.006 & -0.221 & 0 & 0.172 & 0.990 & -0.453 & 0.161 & \\
$(0.172)$ & $(0.069)$ & $(0.006)$ & $(0.113)$ & $(0.054)$ & $(0.035)$ & & $(0.018)$ & $(0.003)$ & & & \\
\hline \hline
\end{tabular}

EGARCH-MN

\begin{tabular}{|c|c|c|c|c|c|c|c|c|c|c|c|}
\hline \hline$\Phi$ & $\mu_{1}$ & $\sigma_{1}$ & $\mu_{2}$ & $\sigma_{2}$ & $\alpha_{0}$ & $\gamma$ & $\alpha_{1}$ & $\beta_{1}$ & $s$ & $r$ & \\
\hline \hline 0.922 & 0.008 & 0.912 & -0.998 & 1.474 & -0.200 & -0.130 & 0.088 & 0.985 & -0.381 & & \\
$(0.006)$ & $(0.002)$ & $(0.251)$ & $(0.091)$ & $(0.281)$ & $(0.056)$ & $(0.018)$ & $(0.023)$ & $(0.004)$ & & & \\
\hline 0.908 & 0.0003 & 0.914 & -0.729 & 1.514 & -0.221 & 0 & 0.171 & 0.990 & -0.453 & 0.159 \\
$(0.015)$ & $(0.000)$ & $(0.321)$ & $(0.109)$ & $(0.360)$ & $(0.041)$ & & $(0.019)$ & $(0.006)$ & & & \\
\hline \hline
\end{tabular}

APARCH-GH

\begin{tabular}{|c|c|c|c|c|c|c|c|c|c|c|c|}
\hline \hline$\alpha$ & $\beta$ & $\delta$ & $\mu$ & $\lambda$ & $\alpha_{0}$ & $\alpha_{1}$ & $\gamma$ & $\beta_{1}$ & $\delta_{\text {vol }}$ & $s$ & $r$ \\
\hline \hline 0.642 & -0.641 & 3.337 & 0.583 & -7.056 & 0.000 & 0.068 & 0.980 & 0.910 & 1.404 & -0.345 & \\
$(0.054)$ & $(0.029)$ & $(0.954)$ & $(0.096)$ & $(1.203)$ & $(0.000)$ & $(0.003)$ & $(0.008)$ & $(0.019)$ & $(0.061)$ & & \\
\hline 1.881 & -0.307 & 1.559 & 0.278 & -0.006 & 0.000 & 0.072 & 0 & 0.918 & 2.180 & -0.402 & 0.140 \\
$(0.254)$ & $(0.075)$ & $(0.421)$ & $(0.032)$ & $(0.001)$ & $(0.000)$ & $(0.011)$ & & $(0.022)$ & $(0.059)$ & & \\
\hline
\end{tabular}

APARCH-MN

\begin{tabular}{|c|c|c|c|c|c|c|c|c|c|c|c|}
\hline \hline$\Phi$ & $\mu_{1}$ & $\sigma_{1}$ & $\mu_{2}$ & $\sigma_{2}$ & $\alpha_{0}$ & $\alpha_{1}$ & $\gamma$ & $\beta_{1}$ & $\delta_{\text {vol }}$ & $s$ & $r$ \\
\hline \hline 0.906 & 0.099 & 0.886 & -0.855 & 1.340 & 0.000 & 0.071 & 0.580 & 0.910 & 1.353 & -0.315 & \\
$(0.016)$ & $(0.009)$ & $(0.213)$ & $(0.021)$ & $(0.213)$ & $(0.000)$ & $(0.007)$ & $(0.027)$ & $(0.007)$ & $(0.165)$ & & \\
\hline 0.989 & 0.003 & 0.977 & -2.33 & 0.171 & 0.000 & 0.059 & 0 & 0.918 & 2.511 & -0.397 & 0.206 \\
$(0.008)$ & $(0.000)$ & $(0.139)$ & $(1.029)$ & $(0.249)$ & $(0.000)$ & $(0.011)$ & & $(0.009)$ & $(0.310)$ & & \\
\hline
\end{tabular}

Table 3: Estimated parameters with and without leverage effect parameter using S\&P 500 data set from January 2, 1999 to July 20, 2011 and their standard errors. $r=\frac{s_{\gamma=0}-s_{\gamma \neq 0}}{s_{\gamma=0}}$ where $s_{\gamma \neq 0}$ and $s_{\gamma=0}$ are the skewness of the residual with and without leverage effect respectively.

EGARCH-GH

\begin{tabular}{|c|c|c|c|c|c|c|c|c|c|c|}
\hline$\alpha$ & $\beta$ & $\delta$ & $\mu$ & $\lambda$ & $\alpha_{0}$ & $\gamma$ & $\alpha_{1}$ & $\beta_{1}$ & $s$ & $r$ \\
\hline $\begin{array}{l}0.265 \\
(0.061)\end{array}$ & $\begin{array}{c}-0.264 \\
(0.051)\end{array}$ & $\begin{array}{l}2.246 \\
(0.319)\end{array}$ & $\begin{array}{l}0.243 \\
(0.070)\end{array}$ & $\begin{array}{c}-3.744 \\
(0.251)\end{array}$ & $\begin{array}{l}-0.215 \\
(0.026)\end{array}$ & $\begin{array}{c}-0.094 \\
(0.009)\end{array}$ & $\begin{array}{l}0.118 \\
(0.011)\end{array}$ & $\begin{array}{l}0.986 \\
(0.002)\end{array}$ & -0.690 & \\
\hline $\begin{array}{l}1.643 \\
(0.215)\end{array}$ & $\begin{array}{c}-0.183 \\
(0.096)\end{array}$ & $\begin{array}{l}0.937 \\
(0.025)\end{array}$ & $\begin{array}{l}0.179 \\
(0.127)\end{array}$ & $\begin{array}{l}0.537 \\
(0.056)\end{array}$ & $\begin{array}{c}-0.192 \\
(0.028)\end{array}$ & 0 & $\begin{array}{l}0.147 \\
(0.013)\end{array}$ & $\begin{array}{l}0.991 \\
(0.002)\end{array}$ & -0.818 & 0.156 \\
\hline \multicolumn{11}{|c|}{ EGARCH-MN } \\
\hline$\Phi$ & $\mu_{1}$ & $\sigma_{1}$ & $\mu_{2}$ & $\sigma_{2}$ & $\alpha_{0}$ & $\gamma$ & $\alpha_{1}$ & $\beta_{1}$ & $s$ & $r$ \\
\hline $\begin{array}{l}0.046 \\
(0.011)\end{array}$ & $\begin{array}{c}-0.932 \\
(0.451)\end{array}$ & $\begin{array}{l}1.954 \\
(0.652)\end{array}$ & $\begin{array}{l}0.044 \\
(0.008)\end{array}$ & $\begin{array}{l}0.882 \\
(0.106)\end{array}$ & $\begin{array}{c}-0.232 \\
(0.028)\end{array}$ & $\begin{array}{c}-0.100 \\
(0.015)\end{array}$ & $\begin{array}{l}0.124 \\
(0.017)\end{array}$ & $\begin{array}{l}0.984 \\
(0.003)\end{array}$ & -0.678 & \\
\hline $\begin{array}{l}0.063 \\
(0.020)\end{array}$ & $\begin{array}{c}-0.715 \\
(0.328)\end{array}$ & $\begin{array}{l}1.962 \\
(0.649)\end{array}$ & $\begin{array}{l}0.050 \\
(0.007)\end{array}$ & $\begin{array}{l}0.876 \\
(0.112)\end{array}$ & $\begin{array}{c}-0.195 \\
(0.021)\end{array}$ & 0 & $\begin{array}{l}0.155 \\
(0.023)\end{array}$ & $\begin{array}{l}0.991 \\
(0.004)\end{array}$ & -0.806 & 0.159 \\
\hline
\end{tabular}

APARCH-GH

\begin{tabular}{|c|c|c|c|c|c|c|c|c|c|c|c|}
\hline \hline$\alpha$ & $\beta$ & $\delta$ & $\mu$ & $\lambda$ & $\alpha_{0}$ & $\alpha_{1}$ & $\gamma$ & $\beta_{1}$ & $\delta_{\text {vol }}$ & $s$ & $r$ \\
\hline \hline 0.270 & -0.269 & 2.316 & 0.253 & -3.846 & 0.000 & 0.069 & 0.813 & 0.922 & 1.200 & -0.637 & \\
$(0.114)$ & $(0.052)$ & $(0.297)$ & $(0.094)$ & $(0.694)$ & $(0.0001)$ & $(0.007)$ & $(0.100)$ & $(0.007)$ & $(0.028)$ & & \\
\hline 0.219 & -0.218 & 2.121 & 0.212 & -3.340 & 0.000 & 0.073 & 0 & 0.934 & 1.514 & -0.797 & 0.201 \\
$(0.026)$ & $(0.171)$ & $(0.382)$ & $(0.059)$ & $(0.612)$ & $(0.000)$ & $(0.007)$ & & $(0.006)$ & $(0.051)$ & & \\
\hline \hline
\end{tabular}

APARCH-MN

\begin{tabular}{|c|c|c|c|c|c|c|c|c|c|c|c|}
\hline \hline$\Phi$ & $\mu_{1}$ & $\sigma_{1}$ & $\mu_{2}$ & $\sigma_{2}$ & $\alpha_{0}$ & $\alpha_{1}$ & $\gamma$ & $\beta_{1}$ & $\delta_{\text {vol }}$ & $s$ & $r$ \\
\hline \hline 0.942 & 0.047 & 0.888 & -0.813 & 1.903 & 0.000 & 0.071 & 0.814 & 0.921 & 1.198 & -0.634 & \\
$(0.003)$ & $(0.017)$ & $(0.524)$ & $(0.126)$ & $(0.692)$ & $(0.000)$ & $(0.007)$ & $(0.091)$ & $(0.006)$ & $(0.096)$ & & \\
\hline 0.924 & 0.056 & 0.863 & -0.687 & 1.898 & 0.000 & 0.095 & 0 & 0.907 & 1.659 & -0.719 & 0.118 \\
$(0.007)$ & $(0.029)$ & $(0.561)$ & $(0.123)$ & $(0.536)$ & $(0.000)$ & $(0.008)$ & & $(0.007)$ & $(0.155)$ & & \\
\hline \hline
\end{tabular}

Table 4: Estimated parameters with and without leverage effect parameter using the S\&P 500 data set from January 2, 1987 to July 20, 2011 and their standard errors. $r=\frac{s_{\gamma=0}-s_{\gamma \neq 0}}{s_{\gamma=0}}$ where $s_{\gamma \neq 0}$ and $s_{\gamma=0}$ are the skewness of the residual with and without leverage effect respectively.

Focusing on the full sample results presented in Table 4, we obtain consistent evidence that the lever- 
age effect only explains a weak part of the returns' skewness. For the four models used in our experiments, the ratio described by equation (12) ranges from around $11 \%$ in the APARCH-MN case to around $20 \%$ in the APARCH-GH case. The EGARCH based models lead to a ratio approximately equal to $16 \%$. These conclusions hold as well when the ratio is computed from subsets of our full sample. In all our experiments, the maximum $r$ is obtained with the APARCH-GH model over a sample of returns covering the 1987-1998 period. In this case $r=37.6 \%$ : our findings thus show that leverage effects only account for a third of returns' skewness. However, we consistently find that the $\gamma$ parameter across models is estimated to be statistically different from zero: there is a leverage effect at work in the dynamics of S\&P 500 returns, but it only accounts for $30 \%$ of the returns's skewness. In order to better measure the extent to which leverage effect statistically matters, we now use in and out of sample likelihood ratio tests.

\subsection{Testing for leverage effects in-sample}

In this part, we test for the in sample significance of leverage effects using the Hansen (1992)'s test presented earlier.

\begin{tabular}{ccccccc}
\hline \hline Data set & \multicolumn{7}{c}{ From January 2,1987 to December 31, 1998 } \\
\hline \hline$M$ & 0 & 1 & 2 & 3 & 4 & 5 \\
\hline EGARCH-GH & 0.177 & 0.212 & 0.181 & 0.157 & 0.138 & 0.137 \\
APARCH-GH & 0.199 & 0.211 & 0.238 & 0.231 & 0.224 & 0.234 \\
EGARCH-MN & 0.174 & 0.164 & 0.150 & 0.162 & 0.179 & 0.178 \\
APARCH-MN & 0.330 & 0.323 & 0.295 & 0.304 & 0.275 & 0.268 \\
\hline \hline Data set & \multicolumn{7}{c}{ From January 2, 1999 to July 20, 2011 } \\
\hline \hline$M$ & 0 & 1 & 2 & 3 & 4 & 5 \\
\hline EGARCH-GH & 0.158 & 0.153 & 0.124 & 0.108 & 0.138 & 0.127 \\
APARCH-GH & 0.151 & 0.125 & 0.111 & 0.122 & 0.136 & 0.108 \\
EGARCH-MN & 0.143 & 0.131 & 0.136 & 0.156 & 0.142 & 0.130 \\
APARCH-MN & 0.108 & 0.101 & 0.082 & 0.094 & 0.092 & 0.098 \\
\hline \hline Data set & \multicolumn{7}{c}{ From January 2, 1987 to July 20,2011} \\
\hline \hline$M$ & 0 & 1 & 2 & 3 & 4 & 5 \\
\hline EGARCH-GH & 0.174 & 0.105 & 0.146 & 0.151 & 0.147 & 0.142 \\
APARCH-GH & 0.098 & 0.091 & 0.060 & 0.092 & 0.096 & 0.090 \\
EGARCH-MN & 0.113 & 0.126 & 0.146 & 0.149 & 0.152 & 0.121 \\
APARCH-MN & 0.095 & 0.077 & 0.093 & 0.090 & 0.098 & 0.106 \\
\hline \hline
\end{tabular}

Table 5: Hansen's test for the estimated models using the S\&P 500 log returns.

This table presents the p-value of the Hansen's test comparing the models with and without leverage effect parameters for the $S \& P 500$ data set. The test reads as follows: for the data set starting from January 2, 1987 to December 31, 1998, for the EGARCH-GH model and for bandwidth M=0, its p-value is 0.177 . This p-value being greater than $5 \%$, the hypothesis $H_{0}$ (absence of leverage effect) is not rejected.

Table 5 presents the results obtained from Hansen (1992)'s test across different values of the $M$ parameter. As for our previous results, we obtain consistent conclusions across the various sets of models used in our experiments: at a 5\% risk level, none of the $\gamma$ estimates are found to be different from zero, whatever the value of $M$. For example, in the case of the EGARCG-MN model with $M=5$, the test p-value is found to be equal to $13 \%$ that is the minimum value for each of the $M$ used in our tests. The lowest p-value obtained is $6 \%$ in the case of the APARCH-GH model with $M=2$. As for our previous results, breaking down our full sample into sub-periods does not change the conclusions raised from our test: the $\gamma$ parameter is found to be statistically equal to zero. Still, the p-values obtained with the 1987-1998 sample are higher than the ones obtained with the 1999-2011 one, probably in relation with the increase in the number of crisis periods in the second sample. We 
now turn out our attention to an out-of-sample analysis of leverage effects.

\subsection{The role of leverage effects in forecasting the density of returns}

If our previous tests consistently highlighted the weak role played by leverage effects to accurately describe the density of returns, it says little regarding its role in forecasting the future density of returns. We confirm these findings obtained using the first two steps by gauging the usefulness of the leverage effect when it comes to forecasting the future density of returns. To do so, we rely on Amisano and Giacomini (2007)'s forecast density test methodology.

\begin{tabular}{|c|c|c|c|c|c|c|c|}
\hline \multicolumn{8}{|c|}{ S\&P 500 index } \\
\hline \multicolumn{8}{|c|}{ EGARCH-GH } \\
\hline Forecast Horizon & 1 & 10 & 20 & 30 & 40 & 50 & 60 \\
\hline Unweighted $\omega_{0}$ & -2.200 & -2.245 & -2.229 & -2.319 & -2.262 & -2.264 & $\overline{-2.244}$ \\
\hline Center $\omega_{1}$ & -1.306 & -1.328 & -1.293 & -1.364 & -1.318 & -1.324 & -1.292 \\
\hline Tails $\omega_{2}$ & -2.594 & -2.656 & -2.647 & -2.739 & -2.678 & -2.677 & -2.670 \\
\hline Right Tail $\omega_{3}$ & -1.829 & -1.780 & -1.806 & -1.864 & -1.831 & -1.804 & -1.854 \\
\hline Left Tail $\omega_{4}$ & -1.725 & -1.787 & -1.776 & -1.839 & -1.804 & -1.821 & -1.768 \\
\hline \multicolumn{8}{|c|}{ APARCH-GH } \\
\hline Forecast Horizon & 1 & 10 & 20 & 30 & 40 & 50 & 60 \\
\hline Unweighted $\omega_{0}$ & 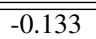 & 0.097 & -0.241 & $\overline{-0.051}$ & 0.099 & 0.284 & $\overline{-0.264}$ \\
\hline Center $\omega_{1}$ & -1.146 & -1.097 & -1.492 & -1.339 & -1.110 & -1.139 & -1.317 \\
\hline Tails $\omega_{2}$ & 0.348 & 0.621 & 0.426 & 0.585 & 0.669 & 0.892 & 0.271 \\
\hline Right Tail $\omega_{3}$ & -0.417 & -0.190 & -0.370 & -0.440 & -0.030 & 0.269 & -0.643 \\
\hline Left Tail $\omega_{4}$ & 0.131 & 0.287 & -0.044 & 0.251 & 0.162 & 0.172 & 0.111 \\
\hline \multicolumn{8}{|c|}{ EGARCH-MN } \\
\hline Forecast Horizon & 1 & 10 & 20 & 30 & 40 & 50 & 60 \\
\hline$\overline{\text { Unweighted } \omega_{0}}$ & "-1.276 & 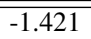 & $\overline{-1.257}$ & $\overline{-1.356}$ & $\overline{-1.436}$ & -1.425 & -1.561 \\
\hline Center $\omega_{1}$ & -1.365 & -1.466 & -1.237 & -1.461 & -1.546 & -1.427 & -1.710 \\
\hline Tails $\omega_{2}$ & -0.786 & -0.931 & -0.879 & -0.814 & -0.879 & -0.958 & -0.932 \\
\hline Right Tail $\omega_{3}$ & 1.326 & 1.283 & 1.414 & 1.251 & 1.051 & 1.274 & 0.973 \\
\hline Left Tail $\omega_{4}$ & -2.029 & -2.125 & -2.018 & -2.129 & -2.067 & -2.192 & -2.179 \\
\hline \multicolumn{8}{|c|}{ APARCH-MN } \\
\hline Forecast Horizon & 1 & 10 & 20 & 30 & 40 & 50 & 60 \\
\hline$\overline{\text { Unweighted } \omega_{0}}$ & 0.493 & 1.289 & 0.786 & 1.330 & 1.047 & 1.070 & 0.778 \\
\hline Center $\omega_{1}$ & 0.514 & 0.856 & 0.447 & 0.828 & 1.032 & 1.308 & 0.482 \\
\hline Tails $\omega_{2}$ & 0.272 & 1.542 & 1.122 & 1.672 & 0.634 & 0.036 & 1.174 \\
\hline Right Tail $\omega_{3}$ & 0.277 & 1.691 & 0.834 & 1.309 & 0.649 & 1.478 & 0.902 \\
\hline Left Tail $\omega_{4}$ & 0.562 & 0.533 & 0.551 & 0.993 & 1.223 & 0.476 & 0.445 \\
\hline
\end{tabular}

Table 6: Amisano Giacomini Test Statistics using S\&P 500 data set.

This table presents Amisano Giacomini's test comparing the models with and without leverage effect parameter using the S\&P 500 data set covering the 1987-2011 period. We use rolling windows of size $\tau=2000$ and a maximal forecast horizon $k_{\max }$ of 60 days. The test reads as follows: for the EGARCH-GH model, for horizon $k=1$ and for the weight $\omega_{0}=1$, the test statistic is -2.200: this value being outside the [-1.96:1.96] 5\% interval confidence, the null hypothesis that both models (with or without leverage) are equivalent is rejected in favor of the non leveraged one (negative value).

Table 6 presents the tests results obtained using a rolling window of 2000 trading days. The first sample ends on May 8, 2003. We use a fixed window scheme rolling from one day to another and reestimating each time each model's parameters using the recursive estimation methodology. Here again, the consistency of our results is significant: for forecasting horizons ranging from 1 to 60 days the models with leverage effects are found to be equivalent to models without leverage effects. This result holds for the different weight functions. For example, in the case of the EGARCH-MN model, when using the $w_{0}$ weight function and for a forecast horizon of 30 days, the Amisano and Giacomini 
(2007)'s test statistics is equal to -1.356: a model with or without leverage effect parameter provides statistically equivalent density forecasts at a $5 \%$ risk level. Now, our results contain three cases for which both models are not found to be statistically equivalent: in the EGARCH-GH case with the $w_{0}$ and $w_{2}$ weights and in the EGARCH-MN case with the $w_{4}$ weight, the model without leverage effects is found to dominate the one with leverage effects. Hence across all our forecasting results, the model without leverage effect is found to be equivalent or better than the one with leverage effect.

Considering our three previous tests and measures, we obtain very consistent results showing that leverage effects do not seem to have a statistical significance when it comes to modeling the returns on US equities as proxied by the S\&P 500 .

\subsection{Testing for leverage effects at a stock level}

This final section investigates the importance of leverage effects at a stock-by-stock level. Black (1976) and Christie (1982) discuss the possibility that leverage effects can impact individual stocks. Stock-level leverage effects are not incompatible with our previous findings: once diversified away through index investing, leverage effects would therefore disappear, explaining the previously listed empirical results. Using the components of the SP500 index, we run the in-sample Hansen test and measure the percentage of stocks for which we find a statistically significant leverage effect ${ }^{6}$.

Our empirical strategy unfolds as follow: first of all, we use the list of stocks available in the 2011 composition of the SP500 index. We discard the stocks for which we have less than 2500 days of market quotes. Then, stock by stock, we run the Hansen (1992) test as detailed in Subsection 3.3.2 for various values for the $M$ bandwidth parameter. Out of the 500 components, our screening for lengthy enough time series led us to make use of 436 stocks. Across those stocks the conclusion from the Hansen (1992) test were consistent across the various values for the bandwidth $M$ which is why Table 7 only shows results for $M=0$.

\begin{tabular}{|l|c|c|c|c|}
\hline \hline Sector & EGARCH-GH & EGARCH-MN & APARCH-MN & APARCH-GH \\
\hline \hline Basic Materials & 86.96 & 76.09 & 69.70 & 82.61 \\
\hline Communications & 68.52 & 77.56 & 85.19 & 67.90 \\
\hline Consumer, Cyclical & 67.23 & 72.92 & 59.89 & 73.16 \\
\hline Consumer, non-cyclical & 77.84 & 66.67 & 58.14 & $76 ; 39$ \\
\hline Energy & 84.21 & 78.95 & 79.82 & 86.75 \\
\hline Financial & 67.76 & 76.22 & 73.02 & 72.59 \\
\hline Industrial & 83.89 & 80.33 & 63.89 & 86.11 \\
\hline Technology & 60 & 70.20 & 71.43 & 64.28 \\
\hline Utilities & 86.67 & 63.70 & 86.67 & 86.11 \\
\hline \hline \% TOTAL & 75.15 & 73.57 & 69.14 & 77.11 \\
\hline \hline
\end{tabular}

Table 7: Percentage of stocks per sector for which leverage is statistically not significant from the Hansen (1992) test

This table displays the percentage of p-values found out to be higher than 5\%, that are the cases for which we statistically discard the null hypothesis of leverage effect. Results are grouped by industry sector as well, so that to monitor whether companies' sector makes a difference in terms of leverage effect. The repartition of stocks within the $S \& P 500$ index is presented in Table 8.

\footnotetext{
${ }^{6}$ Given the numerical cost associated to the Amisano and Giacomini (2007) test and the number of series to be handled we decided to solely rely on the Hansen (1992) test.
} 


\begin{tabular}{|l|c|}
\hline \hline Sector & \% of each sector in the SP500 \\
\hline \hline Basic Materials & 5.26 \\
\hline Communications & 6.18 \\
\hline Consumer, Cyclical & 13.50 \\
\hline Consumer, non-cyclical & 19.45 \\
\hline Energy & 8.92 \\
\hline Financial & 17.62 \\
\hline Industrial & 13.96 \\
\hline Technology & 8.01 \\
\hline Utilities & 6.86 \\
\hline \hline
\end{tabular}

Table 8: Percentage of stocks per sector in the S\&P500 in January 2013

From Table 7, on average we find that for $70 \%$ of the stocks there is no statistically significant leverage effects. This conclusion is both consistent with the conclusions raised from the previous sections based on the index itself and with the suspicion that stocks have a greater tendency to generate a return-to-volatility effect than indices. This conclusion holds across the various model specifications and for most of the sectors. Striking differences remain: we find a higher rate of companies with leverage effects in the "Technology" sector. In the case of the "Energy" and "Utilities" sectors, we find a higher than $70 \%$ figure consistently across models: those two sectors seem to exhibit the most infrequent leverage effects of the sample used here.

\section{4 conclusion}

This article questions the empirical usefulness of leverage effects to describe the dynamics of equity returns. We both test for the statistical significance of leverage effects. Relying on both in- and outof-sample tests, we consistently find a weak contribution of leverage effects over the past 25 years of S\&P 500 returns, casting light on the importance of the conditional distribution in time series models. Digging into the components of the index, we find that this conclusion holds for $70 \%$ of the stocks within: a higher rate of leverage effects is therefore found in individual stocks, while still not dominating the sample used in our experiments.

\section{Appendix: Numerical implementation of the maximum-likelihood esti- mation}

In this appendix we briefly present the numerical implementation of the so-called conditional maximumlikelihood estimation that is used in this paper. We refer the reader to Chorro et al. (2016), Section 2.6.3 for more details.

1. We start from the Quasi-maximum-likelihood estimate $\hat{\theta}_{T}^{1}=\left(\widetilde{\theta_{T}^{D}}, \widetilde{\theta_{T}^{V}}\right)$ of the model given by equations (1) and (2).

2. We re-estimate the volatility parameter $\theta^{V}$ by maximizing

$$
\sum_{t=1}^{T}-\frac{\log \left(h_{t}\right)}{2}+\log \left[d_{\widetilde{\theta_{T}^{D}}}\left(\frac{y_{t}}{\sqrt{h_{t}}}\right)\right],
$$

obtaining $\hat{\theta}_{T}^{2, V}$.

3. We re-estimate the distribution parameter $\theta^{D}$ from the standardized residuals

$$
\left(\frac{y_{1}}{\sqrt{h_{1}}\left(\hat{\theta}_{T}^{2, V}\right)}, \ldots, \frac{y_{T}}{\sqrt{h_{T}}\left(\hat{\theta}_{T}^{2, V}\right)}\right)
$$


by maximizing

$$
\sum_{t=1}^{T} \log \left[d_{\theta^{D}}\left(\frac{y_{t}}{\sqrt{h_{t}}\left(\hat{\theta}_{T}^{2, V}\right)}\right)\right]
$$

and obtain $\hat{\theta}_{T}^{2, D}$ and $\hat{\theta}_{T}^{2}=\left(\hat{\theta}_{T}^{2, D}, \hat{\theta}_{T}^{2, V}\right)$.

4. We iterate this procedure until a good trade off between precision and computational cost is reached. In this paper we use 10 iterations.

\section{References}

[1] Aydemir, A.C., Gallmeyer, M. and Hollifield, B., 2006. Financial Leverage Does Not Cause the Leverage Effect. Working Paper, Carnegie Mellon University.

[2] Akgiray, V., Booth, G.G., 1987. Compound Distribution Models of Stock Returns: An Empirical Comparison. The Journal of Financial Research 10, 269-280.

[3] Alexander, C., Lazar, E., 2006. Normal Mixture GARCH(1,1): Applications to Exchange Rate Modeling. Journal of Applied Econometrics 21, 307-336.

[4] Amisano, G., Giacomini, R., 2007. Comparing Density Forecasts via Weighted Likelihood Ratio Tests. Journal of Business \& Economic Statistics, American Statistical Association 25, 177-190.

[5] Awartani, B.M.A., Corradi, V., 2005. Predicting the volatility of the S\&P -500 stock index via GARCH models: the role of asymmetries. International Journal of Forecasting, 21, 167-183.

[6] Badescu, A., Kulperger, R., Lazar, E., 2008. Option Valuation with Normal Mixture GARCH Models. Studies in Nonlinear Dynamics and Econometrics 12 (2), 1580-1580.

[7] Badescu, A., Elliott, R.J., Kulperger, R., Miettinen, J. and Siu, T.K., 2011. A comparison of pricing kernels for GARCH option pricing with generalized hyperbolic distributions. International Journal of Theoretical and Applied Finance, 14(5), 669-708.

[8] Bae, J., Kim, C.J., and Nelson, C.R., 2007. Why are stock returns and volatility negatively correlated?. Journal of Empirical Finance, 14(1), 41-58.

[9] Barndorff-Nielsen, O.E., 1977. Exponentially decreasing distributions for the logarithm of particle size. Proceedings of the Royal Society of London Series A, 353, 401-419.

[10] Bandi, F.M, Reno, R., 2012. Time varying leverage effects. Journal of Econometrics, 169(1), 94-113.

[11] Bates, D., 1996. Jumps and stochastic volatility: exchange rate processes implicit in deutsche mark options. Review of Financial studies, 9(1), 69-107.

[12] Behboodian, J., 1970. On the Modes of a Mixture of Two Normal Distributions. Technometrics, 12(1), 131-139.

[13] Bekaert, G., Wu, G., 2000. Asymmetric volatility and risk in equity markets. Review of Financial Studies, 13 (1), 1-42.

[14] Black, F., 1976. Studies of stock prices volatility Changes. Proceedings of the 1976 Meetings of the American Statistical Association, Business and Economics Statistics Section, 177-189. 
[15] Campbell, J.Y., Hentschel, L., 1992. No news is good news: An asymmetric model of changing volatility in stock returns. Journal of Financial Economics, 31, 281318.

[16] Chorro, C., Guégan, D., Ielpo, F., 2010. Martingalized Historical approach for Option Pricing. Finance Research Letters 7 (1), 24-28.

[17] Chorro, C., Guégan, D. and Ielpo, F., 2012. Option pricing for GARCH type models with generalized Hyperbolic Innovations. Quantitative Finance, 12(7), 1079-1094.

[18] Chorro,C., Guégan, D. and Ielpo, F., 2016. A Time Series Approach to Option Pricing. Models, Methods and Empirical Performances. Springer-Verlag.

[19] Christie, A.A., 1982. The stochastic behavior of common stock variances. Journal of Financial Economics, 10, 407432.

[20] Christoffersen, P. and Jacobs, K., 2004. Which Garch model for Option valuation. Management science, 50(9), 1204-1221.

[21] Christoffersen, P., Heston, S., Jacobs, K., 2006. Option Valuation with Conditional Skewness. Journal of Econometrics 131, 253-284.

[22] Christoffersen, P., Elkamhi, R., Feunou, B., Jacobs, K., 2010. Option Valuation with Conditional Heteroskedasticity and Non-Normality. Review of Financial studies, 23(5), 2139-2183.

[23] Corsi, F., Reno, R., 2012. Discrete-Time Volatility Forecasting With Persistent Leverage Effect and the Link With Continuous-Time Volatility Modeling. Journal of Business \& Economic Statistics, 30(3), 2012.

[24] Curto, J., Pinto, J., Tavares, G., 2009. Modeling stock markets volatility using GARCH models with normal Students $t$ and stable Paretian distributions. Statistical Papers, 50 (2), 311-321.

[25] Ding Z., Granger, C.W.J., Engle, R.F., 1993. A Long Memory Property of Stock Market Returns and a New Model. Journal of Empirical Finance 1, 83-106.

[26] Duffee, G.R., 1995. Stock returns and volatility: A firm level analysis. Journal of Financial Economics, 37, 399-420.

[27] Fan, Y., Pastorello, S., Renault, E., 2015. Maximisation by Parts in Extremum Estimation. The econometrics journal, 18(2), 147-171.

[28] Figlewski, S., Wang, X., 2000. Is the leverage effect a leverage effect?. Working Paper, New York University.

[29] Giot, P., and Laurent, S., 2004. Modelling daily value-at-risk using realized volatility and ARCH type models. journal of Empirical Finance, 11(3), 379-398.

[30] Guégan, D., Ielpo, F. and Lalaharison, H., 2013. Option Pricing with Discrete Time Jump Processes. Journal of Economic Dynamics and Control, 37 (12), 2417-2445.

[31] Hansen, B.E., 1992. The Likelihood Ratio Test under Nonstandard Conditions: Testing the Markov Switching Model of GNP. Journal of Applied Econometrics, 7(1), 61-82.

[32] Hansen, P. R., Huang, Z., and Shek, H.H., 2012. Realized garch: a joint model for returns and realized measures of volatility. Journal of Applied Econometrics, 27(6), 877-906.

[33] Heston, S.L., 1993. A closed-form solution for options with stochastic volatility, with applications to bond and currency options. The Review of Financial Studies, 6, 327-343. 
[34] Kon, S. J., 1984. Models of Stock Returns: A Comparison. The Journal of Finance 39, 147-165.

[35] Maheu, J.M. and McCurdy, T.H., 2011. Do high-frequency measures of volatility improve forecasts of return distributions? Journal of Econometrics, 160, 69-76.

[36] Nelson, D.B., 1991. Conditional Heteroskedasticity in Asset Returns. Econometrica 59, 347370.

[37] Poon, S. H., Granger, C.W.J., 2003. Forecasting volatility in financial markets: A review. Journal of Economic Literature, 41, 478539.

[38] Schwert, G.W., 1989. Why does stock market volatility change over time?. Journal of Finance, $44,1115-1153$.

[39] Tucker, A. L., Pond, L., 1998. The Probability Distribution of Foreign Exchange Price Changes: Tests of Candidate Processes. Review of Economics and Statistics, 11, 638-647.

[40] Wang, K. L., Fawson, C., Barrett, C. B., and McDonald, J. B., 2001. A flexible parametric GARCH model with an application to exchange rates. Journal of Applied Econometrics, 16(4), 521-536.

[41] Wu, G., 2001. The determinants of asymmetric volatility. Review of Financial Studies, 14, 837 859. 